\title{
IRREDUCIBLE REPRESENTATIONS OF THE ALTERNATING GROUP IN ODD CHARACTERISTIC
}

\author{
BEN FORD
}

(Communicated by Ronald M. Solomon)

\begin{abstract}
We use the recently-proved conjecture of Mullineux to determine which modular irreducible representations of the symmetric group $\Sigma_{n}$ split on restriction to $A_{n}$, and which remain irreducible (everything taking place over a splitting field for $A_{n}$ of characteristic $p>2$ ). An indexing of the absolutely irreducible representations of $A_{n}$ is thus obtained. A modular analogue of the Frobenius symbol for a partition is introduced, which makes the Mullineux map somewhat more intuitive.
\end{abstract}

\section{INTRODUCTION AND NOTATION}

Parameterizations of the irreducible representations of a class of groups by some combinatorial objects provide some of the most appealing results and useful tools in group theory - labeled Dynkin diagrams (groups of Lie type), partitions and tableaux (symmetric groups), etc. It is Alfred Young's parameterization of the ordinary representations of the symmetric group $\Sigma_{n}$ by partitions of $n$ (see [9] for an overview) that began the line of research we continue here. In particular, Young's constructions were extended by James ([6]) to representations of $\Sigma_{n}$ over an arbitrary field $K$ of characteristic $p$, and we investigate these modular representations which James constructed.

As soon as one has a result about the symmetric group, it is natural to ask what the result implies for the alternating group $A_{n}$. Young's constructions can be used to give a nice parameterization of the ordinary irreducible representations of the alternating group $A_{n}$ by describing those irreducible representations of $\Sigma_{n}$ which split upon restriction to $A_{n}$ (see $[7, \S 2.5]$ ). But James's parameterization of the modular irreducible representations of $\Sigma_{n}$ has proved much more difficult to use to obtain information about $A_{n}$. Benson in [3] used a reduction modulo two of the spin representations to determine which 2-modular irreducible representations of $\Sigma_{n}$ are irreducible upon restriction to $A_{n}$, and which split as the sum of two (non-isomorphic) irreducible modules (that each $\Sigma_{n}$-module must do one of these things is clear as $A_{n}$ is normal of index 2 in $\Sigma_{n}$ ).

With the Mullineux conjecture ([8]) in the representation theory of the symmetric group now proved ([5]), a reasonably nice parameterization of the absolutely

Received by the editors August 28, 1995.

1991 Mathematics Subject Classification. Primary 20C20, 20C30.

Supported in part by the NSA.

Thanks to Jens C. Jantzen for suggesting this question. 
irreducible representations of the alternating group $A_{n}$ over a field of odd characteristic is now available. We will assume that $K$ is a splitting field for $A_{n}(n>1)$, of characteristic $p>2$.

The standard approach in descending from $\Sigma_{n}$ to $A_{n}$ is to use Clifford's theory (see Curtis and Reiner $[4, \S 49]$ ), of which we need only an easy application since $\Sigma_{n} / A_{n}$ has order 2 .

First some notation: Let $\lambda$ be a partition of $n$ (written $\lambda \vdash n$ ); that is,

$$
\lambda=\left(l_{1} \geq l_{2} \geq \cdots \geq l_{m}>0\right), \quad \sum l_{i}=n .
$$

We usually collect like parts together and write

$$
\lambda=\left(\lambda_{1}^{a_{1}}, \ldots, \lambda_{k}^{a_{k}}\right),
$$

with $\lambda_{i}>\lambda_{i+1}>0$ for $i=1, \ldots, k-1 ; a_{i} \neq 0$; and $\sum a_{i} \lambda_{i}=n$. That the number of partitions of $n$ is equal to the number of possible cycle structures for an element of $\Sigma_{n}$, and thus to the number of conjugacy classes in $\Sigma_{n}$, is clear. But the number of conjugacy classes in $\Sigma_{n}$ is the same as the number of ordinary irreducible representations. Young showed how to construct (over $\mathbf{Q}$ ) an irreducible module $S^{\lambda}$ for each partition $\lambda$, such that if $\mu$ and $\lambda$ are distinct partitions, then $S^{\lambda}$ and $S^{\mu}$ are not isomorphic.

We use the symbol $[\lambda]$ to represent the partition's Young diagram, which consists of $n$ nodes $\bullet$ placed in decreasing rows, as illustrated by the following example. Let $\lambda=\left(5^{2}, 3,1\right)$ (so $\left.n=14\right)$; then the Young diagram is:

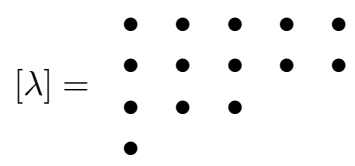

By the rim of $[\lambda]$ we shall mean the collection of nodes which are either at the bottom of a column, at the right end of a row, or both.

Given a partition $\lambda$, one obtains another partition $\lambda^{\prime}$, called the conjugate partition to $\lambda$, by transposing the Young diagram $[\lambda]$ about its main diagonal. So if $\lambda=\left(5^{2}, 3,1\right)$ as above, then the Young diagram for $\lambda^{\prime}$ is

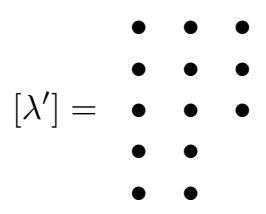

and $\lambda^{\prime}=\left(4,3^{2}, 2^{2}\right)$.

Finally, If $H$ is a subgroup of a group $G$, and $M$ is an $F G$-module for some field $F$, by $M \downarrow_{H}$ we shall mean $M$ restricted to $H$, that is, $M$ considered as a module over $F H \subseteq F G$. If $N$ is an $F H$-module, then $N \uparrow^{G}$ will be the induced module $F G \otimes_{F H} N$ (see $[4, \S 38]$ for more).

\section{Mullineux: A modular $\lambda^{\prime}$}

Of course, if $\lambda$ is a partition of $n$, then so is $\lambda^{\prime}$, and so $\lambda$ and $\lambda^{\prime}$ correspond to (perhaps different, if $\lambda \neq \lambda^{\prime}$ ) irreducible $\mathbf{Q}$-representations of $\Sigma_{n}$. The crucial fact in descending to $A_{n}$ is that $S^{\lambda} \otimes \operatorname{sgn}_{n}=S^{\lambda^{\prime}}$, where for $\sigma \in \Sigma_{n}, \operatorname{sgn}_{n}(\sigma)=1$ if $\sigma$ is an even permutation, and $\operatorname{sgn}_{n}(\sigma)=-1$ if $\sigma$ is odd. Thus $S^{\lambda} \downarrow_{A_{n}}=S^{\lambda^{\prime}} \downarrow_{A_{n}}$ (as 
the two representations differ only on odd elements of $\Sigma_{n}$ ). Clifford's theory then allows us to tell fairly easily when $S^{\lambda} \downarrow_{A_{n}}$ is irreducible (this happens if $\lambda \neq \lambda^{\prime}$ ), and when $S^{\lambda} \downarrow_{A_{n}}$ is the sum of two inequivalent irreducible $A_{n}$-modules (if $\lambda=\lambda^{\prime}$ ).

If we now consider representations over $K$ instead of over $\mathbf{Q}$, things don't work as nicely. Assume $\lambda$ is a $p$-regular partition, and let $D^{\lambda}$ be the irreducible $K \Sigma_{n^{-}}$ module corresponding to $\lambda$. It is still true that $\left(D^{\lambda} \otimes \operatorname{sgn}_{n}\right) \downarrow_{A_{n}}=D^{\lambda} \downarrow_{A_{n}}$, but it is no longer true that $D^{\lambda} \otimes \operatorname{sgn}_{n}=D^{\lambda^{\prime}}$. Mullineux's algorithm helps out here and gives a modular analogue of $\lambda^{\prime}$. That is, Mullineux in [8] defined a bijection $m$ on the set of $p$-regular partitions of $n$, and his conjecture that $D^{\lambda} \otimes \operatorname{sgn}_{n}=D^{m(\lambda)}$ has now been verified ([5]). With this in hand, we can describe the irreducible $K$-representations of $A_{n}$.

Clearly $D^{\lambda} \downarrow_{A_{n}}=D^{m(\lambda)} \downarrow_{A_{n}}$, as in the characteristic zero case. Also, $D^{\lambda} \downarrow_{A_{n}}$ is completely reducible (since $A_{n}$ is a normal subgroup of $\Sigma_{n}$ ). Consider an irreducible summand $D$ of $D^{\lambda} \downarrow_{A_{n}}\left(=D^{m(\lambda)} \downarrow_{A_{n}}\right)$. If we consider the action of an odd element of $\Sigma_{n}$, say (12), on $D$, the possibilities are that $D(12)=D$ (the inertia group of $D$ is $\Sigma_{n}$ ), or $D(12) \neq D$ (the inertia group of $D$ is $A_{n}$ ).

In the first case, we have that $D=D^{\lambda} \downarrow_{A_{n}}$ is irreducible, since $D^{\lambda}$ is an irreducible $\Sigma_{n}$-module and $D$ is an invariant submodule. Then it is easy to see that $D^{\lambda}$ and $D^{\lambda} \otimes \operatorname{sgn}_{n}=D^{m(\lambda)}$ are not isomorphic as $\Sigma_{n}$-modules: Assume that $\sigma$ is an $\Sigma_{n}$-isomorphism. Then $\sigma$ is also an $A_{n}$-isomorphism; but $D$ is an irreducible $A_{n}$-module and $K$ is a splitting field for $A_{n}$. Thus $\sigma$ must be a scalar multiple of the identity map; but this contradicts the assumption that it is an isomorphism between $D^{\lambda}$ and $D^{\lambda} \otimes \operatorname{sgn}_{n}$.

If, on the other hand, $D(12) \neq D$, then Clifford's theorem says

$$
D^{\lambda} \downarrow_{A_{n}}=D \oplus D(12)=D^{m(\lambda)} \downarrow_{A_{n}},
$$

and this implies (by Mackey's subgroup theorem; see for example $[4, \S 44]$ ) that $D^{\lambda} \cong D \uparrow^{\Sigma_{n}} \cong D^{m(\lambda)}$.

Summarizing, we have:

Theorem 2.1. 1. If $\lambda \neq m(\lambda)$, then $D^{\lambda} \downarrow_{A_{n}}$ is irreducible.

2. If $\lambda=m(\lambda)$, then $D^{\lambda} \downarrow_{A_{n}}$ is the sum of two irreducible, non-equivalent, representations of $A_{n}$, say $D_{+}^{\lambda}$ and $D_{-}^{\lambda}$.

Finally,

$$
\left\{D^{\lambda} \downarrow_{A_{n}} \mid \lambda \neq m(\lambda)\right\} \cup\left\{D_{+}^{\lambda}, D_{-}^{\lambda} \mid \lambda=m(\lambda)\right\}
$$

is a complete system of inequivalent irreducible $K A_{n}$-modules.

\section{Fixed points And a modular Frobenius symbol}

So the issue is identifying the fixed points of the Mullineux map. Andrews and Olsson in [2] counted the fixed points of $m$ and described them to some extent. Unfortunately, the fixed points are not easy to "see" from the diagram or the usual presentation of the partitions, as they are in the ordinary case. We shall describe (briefly) the Mullineux map and describe its fixed points. We will use an alternative method of recording the data involved, which makes the map a bit more appealing.

First let us recall the Frobenius symbol of a partition (see for example [1]), a method of representing a partition to make the conjugation action obvious. Given 
a partition $\lambda$, we draw its Young diagram $[\lambda]$, and then construct a $2 \times k$ array

$$
\operatorname{Fr}(\lambda)=\left(\begin{array}{llll}
a_{1} & a_{2} & \cdots & a_{k} \\
b_{1} & b_{2} & \cdots & b_{k}
\end{array}\right),
$$

where $k$ is the number of nodes on the main diagonal of the diagram, $a_{i}$ is the number of nodes to the right of the $i$ th node on the diagonal, and $b_{i}$ is the number of nodes below the $i$ th node on the diagonal. For example, the Frobenius symbol for $\lambda=\left(5^{2}, 3,1\right)$ is $\operatorname{Fr}(\lambda)=\left(\begin{array}{lll}4 & 3 & 0 \\ 3 & 1 & 0\end{array}\right)$, as we see from the diagram below.

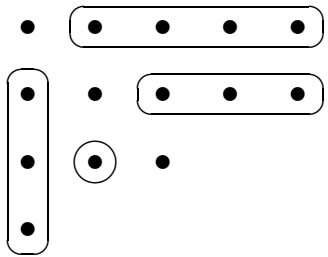

Notice that $n$ is the sum of all the entries in the array, plus the number of columns. Also notice that another way to obtain the symbol is to set

$$
\begin{aligned}
& a_{1}=(\text { the length of the rim of }[\lambda])-(\text { the number of rows in }[\lambda]), \\
& b_{1}=(\text { the number of rows in }[\lambda])-1 ;
\end{aligned}
$$

then remove the rim and repeat to obtain $a_{2}, b_{2}$, etc.

The advantage of the notation is that $\operatorname{Fr}\left(\lambda^{\prime}\right)$ is obtained from $\operatorname{Fr}(\lambda)$ by simply interchanging the two rows. In particular, the fixed points of the conjugation map become obvious. We shall see that with an appropriately defined modular analogue of $\operatorname{Fr}(\lambda)$, the same is true of the Mullineux map.

Given a partition $\lambda$, we define the $p$-rim of the associated Young diagram $[\lambda]$ as follows (see [5] for a more rigorous definition): Beginning at the top right-hand corner of $[\lambda]$, the first $p$ nodes of the rim are in the $p$-rim. Then skip to the next row, and take the next $p$ nodes of the rim. Continue until we reach the end of the rim; the last of these " $p$-segments" may contain fewer than $p$ nodes. For example, the 3 -rim and the 5 -rim of the diagram for $\lambda=\left(5^{2}, 3,1\right)$ are shown as open circles:

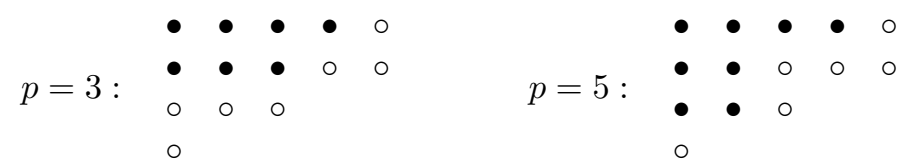

Now let $h_{1}$ be the number of nodes in the $p$-rim of $\lambda$, and let $r_{1}$ be the number of rows in $\lambda$. Delete the $p$-rim and repeat to obtain sequences $h_{1}, h_{2}, \ldots$ and $r_{1}, r_{2}, \ldots$ Let $k$ be such that $h_{k+1}=r_{k+1}=0$, but $h_{k} \neq 0 \neq r_{k}$. The standard way of keeping track of this data is via the Mullineux symbol

$$
M(\lambda)=\left(\begin{array}{llll}
h_{1} & h_{2} & \cdots & h_{k} \\
r_{1} & r_{2} & \cdots & r_{k}
\end{array}\right) ;
$$

but we want to give a different formulation which encodes the same information but makes the desired map more intuitive.

The $p$-modular Frobenius symbol for $\lambda, \operatorname{Fr}_{p}(\lambda)$, will be a $3 \times k$ array

$$
\operatorname{Fr}_{p}(\lambda)=\left(\begin{array}{cccc}
a_{1} & a_{2} & \cdots & a_{k} \\
b_{1} & b_{2} & \cdots & b_{k} \\
\varepsilon_{1} & \varepsilon_{2} & \cdots & \varepsilon_{k}
\end{array}\right)
$$


constructed as follows:

$$
\begin{aligned}
& \varepsilon_{i}=\left\{\begin{array}{l}
0 \text { if } p \mid h_{i}, \\
1 \text { if } p \nmid h_{i},
\end{array}\right. \\
& a_{i}=h_{i}-r_{i}, \\
& b_{i}=r_{i}-\varepsilon_{i} .
\end{aligned}
$$

Notice that $n$ is the sum of all the entries in $\operatorname{Fr}_{p}(\lambda)$.

The fact that the Mullineux symbol uniquely determines $\lambda$ (proved by Mullineux in [8]) means that the modular Frobenius symbol uniquely determines $\lambda$ (as it is possible to reconstruct $M(\lambda)$ from $\operatorname{Fr}_{p}(\lambda)$ ).

Finally, if $\lambda$ has modular Frobenius symbol

$$
\operatorname{Fr}_{p}(\lambda)=\left(\begin{array}{cccc}
a_{1} & a_{2} & \cdots & a_{k} \\
b_{1} & b_{2} & \cdots & b_{k} \\
\varepsilon_{1} & \varepsilon_{2} & \cdots & \varepsilon_{k}
\end{array}\right)
$$

then the Mullineux map $m$ is defined by

$$
\operatorname{Fr}_{p}(m(\lambda))=\left(\begin{array}{cccc}
b_{1} & b_{2} & \cdots & b_{k} \\
a_{1} & a_{2} & \cdots & a_{k} \\
\varepsilon_{1} & \varepsilon_{2} & \cdots & \varepsilon_{k}
\end{array}\right)
$$

So to perform the $p$-analogue of conjugation, we just interchange the top two rows of the modular Frobenius symbol for the partition - just as in the ordinary case, we switch the two rows of $\operatorname{Fr}(\lambda)$.

Now it is easy to see the fixed points of $m: \lambda=m(\lambda)$ if and only if the top two rows of $\operatorname{Fr}_{p}(\lambda)$ are the same. For example, if $\lambda=\left(5^{2}, 3,1\right)$, then

$$
\operatorname{Fr}_{3}(\lambda)=\left(\begin{array}{cccc}
3 & 1 & 1 & 0 \\
3 & 1 & 1 & 0 \\
1 & 1 & 1 & 1
\end{array}\right) \quad \operatorname{Fr}_{5}(\lambda)=\left(\begin{array}{ccc}
2 & 2 & 0 \\
3 & 3 & 2 \\
1 & 0 & 1
\end{array}\right)
$$

Thus if char $K=3, D^{\lambda} \cong D^{m(\lambda)}$ and thus $D^{\lambda} \downarrow_{A_{n}}$ splits as a sum of two irreducible modules; while if char $K=5, D^{\lambda} \neq D^{m(\lambda)}$, and so $D^{\lambda} \downarrow_{A_{n}}$ is irreducible.

\section{REFERENCES}

[1] George E. Andrews, Generalized Frobenius partitions, Mem. Amer. Math. Soc. 49 (1984), no. 301, 1-44. MR 85m:11063

[2] George E. Andrews and Jørn B. Olsson, Partition identities with an application to group representation theory, J. Reine Angew. Math. 413 (1991), 198-212. MR 91k:20019

[3] Dave Benson, Spin modules for symmetric groups, J. London Math. Soc. (2) 38 (1988), 250262. MR 89k:20020

[4] Charles W. Curtis and Irving Reiner, Representation theory of finite groups and associative algebras, Wiley, New York, 1962. MR 26:2519

[5] Ben Ford and Alexander Kleshchev, A proof of the Mullineux conjecture, Math. Z. (to appear).

[6] Gordon D. James, The irreducible representations of the symmetric groups, Bull. London Math. Soc. 8 (1976), 229-232. MR 54:5329

[7] Gordon D. James and Adalbert Kerber, The representation theory of the symmetric group, Addison-Wesley, London, 1981. MR 83k:20003 
[8] Glen Mullineux, Bijections of p-regular partitions and p-modular irreducibles of the symmetric groups, J. London Math. Soc. (2) 20 (1979), 60-66. MR 80j:20016

[9] Daniel Edwin Rutherford, Substitutional analysis, Edinburgh University Press, 1948. MR 10:280i

Department of Mathematics, University of Washington, Box 354350, Seattle, WashINGTON 98195-4350

E-mail address: ford@math.washington.edu

Current address: Department of Mathematics, Case Western Reserve University, Cleveland, Ohio 44106

E-mail address: bjf6@po.cwru.edu 